GLASNIK MATEMATIČKI

Vol. 39(59)(2004), $315-328$

\title{
A NOTE ON EXTENSIONS OF HILBERT C*-MODULES AND THEIR MORPHISMS
}

\author{
Franka Miriam BrüCKLER \\ University of Zagreb, Croatia
}

\begin{abstract}
The aim of this paper is to connect the results of D. Bakic and B. Guljaš about $\mathrm{C}^{*}$-extensions of Hilbert $\mathrm{C}^{*}$-modules with results of D.P. Blecher about Hilbert $\mathrm{C}^{*}$-extensions of operator spaces. In the first part, we give conditions on a completely bounded linear operator between Hilbert $\mathrm{C}^{*}$-modules for the possibility of extending the operator to a "corner-preserving" $\mathrm{C}^{*}$-morphism of the corresponding linking-algebras (or, equivalently, for the operator being a Hilbert $\mathrm{C}^{*}$-morphism). The second part provides an order preserving bijection between the sets of $\mathrm{C}^{*}$ extensions of a Hilbert $\mathrm{C}^{*}$-module and its Hilbert $\mathrm{C}^{*}$-extensions, the latter being a generalized version of Blecher's Hilbert $\mathrm{C}^{*}$-extensions of operator spaces defined in [5].
\end{abstract}

\section{Introduction}

The concept of a Hilbert $\mathrm{C}^{*}$-module is a generalization of the notion of a Hilbert space. The first use of such objects was made by I. Kaplansky in 1953 ([8]). The research on Hilbert $\mathrm{C}^{*}$-modules began in the 70es (W.L. Paschke, [10]; M.A. Rieffel, [12]). A complex vector space $V$ which is a (right) algebraic module over a $\mathrm{C}^{*}$-algebra $\mathcal{A}$ is a Hilbert $\mathcal{A}$-module if there is a map (inner product) $\langle. \mid\rangle:. V \times V \rightarrow \mathcal{A}$ with the properties

$$
\begin{aligned}
\langle x+y \mid z\rangle & =\langle x \mid z\rangle+\langle y \mid z\rangle, \\
\langle x \mid y a\rangle & =\langle x \mid y\rangle a, \\
\langle x \mid y\rangle^{*} & =\langle y \mid x\rangle, \\
\langle x \mid x\rangle & \geq 0 \\
\langle x \mid x\rangle & =0 \Leftrightarrow x=0
\end{aligned}
$$

2000 Mathematics Subject Classification. 46L08, 46L07.

Key words and phrases. Hilbert $\mathrm{C}^{*}$-module, operator space, extension, multiplier algebra, completely bounded map. 
(for all $x, y, z \in V, a \in \mathcal{A}$ ) and such that $V$ is complete with respect to the norm defined by $\|x\|=\sqrt{\|\langle x \mid x\rangle\|_{\mathcal{A}}}$. The simplest example of a Hilbert $\mathcal{A}$-module is the $\mathrm{C}^{*}$-algebra $\mathcal{A}$ with the inner product $\langle a \mid b\rangle=a^{*} b$. Two classes of $(\mathcal{A})$-linear operators on $V$ shall be considered in this paper: the $\mathrm{C}^{*}$ algebra $\mathbf{B}_{\mathcal{A}}(V)$ of adjointable maps and the $\mathrm{C}^{*}$-algebra $\mathbf{K}_{\mathcal{A}}(V)$ of generalized compact operators ${ }^{1}$ (the norm-closure of the linear span of all operators $F_{x, y}$, $x, y \in V$, where $\left.F_{x, y}(z)=x\langle y \mid z\rangle\right) . \mathbf{K}_{\mathcal{A}}(V)$ is an ideal in $\mathbf{B}_{\mathcal{A}}(V)$. For more details on Hilbert $\mathrm{C}^{*}$-modules see [9].

A Hilbert $\mathcal{A}$-module $V$ is said to be full if the linear span of all elements $\langle x \mid y\rangle(x, y \in V)$ is dense in $\mathcal{A}$. The closure of the mentioned linear span is denoted $\langle V \mid V\rangle$ and it is always a (closed, twosided) ideal in $\mathcal{A}$. In most cases it is possible to restrict the considerations to full Hilbert $\mathrm{C}^{*}$-modules.

Denote by $V^{*}$ the formal set $\left\{x^{*}: x \in V\right\}$. Provided with the operations $x^{*}+y^{*}=(x+y)^{*}, a x^{*}=\left(x a^{*}\right)^{*}$ and $\left\langle x^{*} \mid y^{*}\right\rangle=\langle x \mid y\rangle, V^{*}$ becomes a left Hilbert $\mathcal{A}$-module. The linking-algebra of a Hilbert $\mathcal{A}$-module $V$ is the set

$$
\mathcal{L}_{V}=\left\{\left[\begin{array}{cc}
K & x \\
y^{*} & a
\end{array}\right]: a \in \mathcal{A}, K \in \mathbf{K}_{\mathcal{A}}(V), x, y \in V\right\},
$$

provided with a $*$-algebra structure in the natural way. It is also a $\mathrm{C}^{*}$-algebra (see [7]) and the embeddings of $V$ and $\mathcal{A}$ and $\mathbf{K}_{\mathcal{A}}(V)$ in $\mathcal{L}_{V}$ are completely isometric (the last two embeddings are in fact $\mathrm{C}^{*}$-monomorphisms). For details on operator spaces, completely bounded maps and complete isomorphisms, see e.g. [11]. The linking-algebra has many uses, and one of its main advantages is that it carries all important information about the module, but provides easier computations: the abstract inner product on $V$ becomes the usual inner product on a $\mathrm{C}^{*}$-algebra:

$$
\left[\begin{array}{cc}
0 & 0 \\
0 & \langle x \mid y\rangle
\end{array}\right]=\left[\begin{array}{ll}
0 & x \\
0 & 0
\end{array}\right]^{*}\left[\begin{array}{ll}
0 & y \\
0 & 0
\end{array}\right] \text {. }
$$

If $\mathcal{I}$ is a (closed, twosided) ideal in $\mathcal{A}$, then the set

$$
V_{\mathcal{I}}=\overline{[\{x a: x \in V, a \in \mathcal{I}\}}
$$

is called an ideal submodule of $V$. Cohen's factorization theorem implies that

$$
V_{\mathcal{I}}=\{x a: x \in V, a \in \mathcal{I}\} .
$$

One of the advantages of regarding only ideal submodules as subobjects of Hilbert $\mathrm{C}^{*}$-modules is the possibility of constructing quotients: the algebraic quotient of a Hilbert $\mathrm{C}^{*}$-module over a submodule can be provided with a natural Hilbert $\mathrm{C}^{*}$-module structure only if the submodule is an ideal one. It is also known that to an (essential) ideal submodule $V_{I}$ of $V$ corresponds the linking algebra $\mathcal{L}_{I}$ which is an (essential) ideal in $\mathcal{L}_{V}$. For more details on ideal modules see [1].

\footnotetext{
${ }^{1}$ The elements of $\mathbf{K}_{\mathcal{A}}(V)$ are not compact in general, but they are constructed from $V$ in the same way as compact operators on a Hilbert space are constructed.
} 
An appropriate class of morphisms for the category of Hilbert $\mathrm{C}^{*}$-modules over possibly different $\mathrm{C}^{*}$-algebras turned out to be the class of all linear operators supported by a $\mathrm{C}^{*}$-morphism: if $V$ is a Hilbert $\mathcal{A}$-module and $W$ is a Hilbert $\mathcal{B}$-module the linear operator $\phi: V \rightarrow W$ is a Hilbert $\mathrm{C}^{*}$-morphism if there is a $\mathrm{C}^{*}$-morphism $\varphi: \mathcal{A} \rightarrow \mathcal{B}$ with the properties

$$
\begin{gathered}
\phi(x a)=\phi(x) \varphi(a) \\
\langle\phi(x) \mid \phi(y)\rangle=\varphi(\langle x \mid y\rangle)
\end{gathered}
$$

for all $x, y \in V, a \in \mathcal{A}$. We will also call such a morphism a $\varphi$-morphism. In fact, it is only necessary to require the second property, since the first is an easy consequence. It is easily shown that every Hilbert $\mathrm{C}^{*}$-morphism is a complete contraction. In [1] it is shown that a $\varphi$-morphism is isometric ${ }^{2}$ if $\varphi$ is injective and if $V$ is full and $\phi$ is injective, then $\varphi$ is injective.

It is a natural question if there is a natural, "corner-preserving", extension of such a morphism between two $\mathrm{C}^{*}$-modules to a $\mathrm{C}^{*}$-morphism between the corresponding linking algebras, i.e. if for such a $\phi$ exists a $\mathrm{C}^{*}$-morphism $\Phi: \mathcal{L}_{V} \rightarrow \mathcal{L}_{W}$ of the form $\Phi=\left[\begin{array}{cc}\tilde{\varphi} & \phi \\ \phi^{*} & \varphi\end{array}\right]$ where $\tilde{\varphi}: \mathbf{K}_{\mathcal{A}}(V) \rightarrow \mathbf{K}_{\mathcal{B}}(W)$ is also a $\mathrm{C}^{*}$-morphism. In the first part of this paper it is shown that such an extension is possible for every Hilbert $\mathrm{C}^{*}$-morphism (with domain a full module) and also a more general result: every completely bounded operator between Hilbert $\mathrm{C}^{*}$-modules satisfying a natural condition (which is automatic for a Hilbert $\mathrm{C}^{*}$-morphism) can be extended to a $\mathrm{C}^{*}$-morphism of the corresponding linking algebras.

It is well known that the multiplier algebra $\mathcal{M}(\mathcal{A})$ of a $\mathrm{C}^{*}$-algebra $\mathcal{A}$ can be realized as the strict completion of $\mathcal{A}$. D. Bakić and B. Guljaš have generalized this concept for Hilbert $\mathrm{C}^{*}$-modules (for details, see [2]), by introducing the strict topology on a Hilbert $\mathrm{C}^{*}$-module induced by an ideal submodule $V$. A strict completion of a (full) Hilbert $\mathcal{A}$-module $V$ is a Hilbert $\mathcal{B}$-module $W$ which is $V$-strictly complete and such that $\mathcal{A}$ is an essential ideal in $\mathcal{B}$. It is proven in [2] that the strict completion of a Hilbert $\mathcal{A}$-module $V$ is (up to isomorphism) the Hilbert $\mathcal{M}(\mathcal{A})$-module $\mathcal{M}(V)=\mathbf{B}(\mathcal{A}, V)$ (consisting of all adjointable maps from $\mathcal{A}$ to $V)$. When $V=\mathcal{A}$, then $\mathcal{M}(V)=\mathcal{M}(\mathcal{A})$.

For a full Hilbert $\mathcal{A}$-module $V$ we define its $\mathrm{C}^{*}$-extension as a quadruple $(W, \mathcal{B}, \phi, \varphi)$ such that $W$ is a Hilbert $\mathcal{B}$-module, $\varphi: \mathcal{A} \rightarrow \mathcal{B}$ is an injective $\mathrm{C}^{*}$-morphism with the image $\operatorname{Im}(\varphi)$ an ideal in $\mathcal{B}, \phi: V \rightarrow W$ a $\varphi$-morphism and $\operatorname{Im}(\phi)=W_{\operatorname{Im}(\varphi)}$. If $\operatorname{Im}(\varphi)$ is an essential ideal in $\mathcal{B}$, we speak of essential extensions. The above mentioned strict completion is an $\mathrm{C}^{*}$-extension (using natural maps $\gamma: \mathcal{A} \rightarrow \mathcal{M}(\mathcal{A})$ and $\Gamma: V \rightarrow \mathcal{M}(V))$ and it is shown in [2] that it is the maximal $\mathrm{C}^{*}$-extension of $V$. The second part of this paper is an attempt of providing a connection between this concept of $\mathrm{C}^{*}$-extensions

\footnotetext{
${ }^{2}$ In fact, $\phi$ will be completely isometric.
} 
of a Hilbert $\mathrm{C}^{*}$-module and of Hilbert $\mathrm{C}^{*}$-extensions Hilbert $\mathrm{C}^{*}$-extensions of operator spaces defined in [5].

\section{Extending operators Between Hilbert $\mathrm{C}^{*}$-modules to $\mathrm{C}^{*}$-MORPHISMS OF LINKING-ALGEBRAS}

As is described in the introduction, Hilbert $\mathrm{C}^{*}$-morphisms are operators between Hilbert $\mathrm{C}^{*}$-modules supported by a $\mathrm{C}^{*}$-morphism of the corresponding $\mathrm{C}^{*}$-algebras. As one can expect, there is a natural way to extend such a morphism to a $\mathrm{C}^{*}$-morphism of the corresponding linking-algebras.

Proposition 2.1. Let $V$ be a full Hilbert $\mathcal{A}$-module and $W$ a Hilbert $\mathcal{B}$ module. If $\varphi: \mathcal{A} \rightarrow \mathcal{B}$ is a $C^{*}$-morphism and $\phi: V \rightarrow W$ is a $\varphi$-morphism, then there are unique $C^{*}$-morphisms $\tilde{\varphi}: \mathbf{K}_{\mathcal{A}}(V) \rightarrow \mathbf{K}_{\mathcal{B}}(W)$ and $\Phi: \mathcal{L}_{V} \rightarrow \mathcal{L}_{W}$ such that

for all $x, y \in V$ and

$$
\tilde{\varphi}\left(F_{x, y}\right)=F_{\phi(x), \phi(y)}
$$

$$
\Phi\left[\begin{array}{cc}
K & x \\
y^{*} & a
\end{array}\right]=\left[\begin{array}{cc}
\tilde{\varphi}(K) & \phi(x) \\
\phi^{*}\left(y^{*}\right) & \varphi(a)
\end{array}\right]
$$

for all $K \in \mathbf{K}_{\mathcal{A}}(V), a \in \mathcal{A}, x, y \in V$.

Proof. First we check that by $\tilde{\varphi}\left(F_{x, y}\right)=F_{\phi(x), \phi(y)}$ we have well defined a $\mathrm{C}^{*}$-morphism $\tilde{\varphi}: \mathbf{K}_{\mathcal{A}}(V) \rightarrow \mathbf{K}_{\mathcal{B}}(W)$. We shall use the known fact that (see [4]) that $\mathbf{K}_{\mathcal{A}}(V)=V \otimes_{h \mathcal{A}} V^{*}$ (completely isometrically, with respect to the isomorphism defined by $\left.x \otimes_{\mathcal{A}} y^{*} \mapsto F_{x, y}\right)$. By $\left(x, y^{*}\right) \mapsto F_{\phi(x), \phi(y)}$ a bilinear operator $V \times V^{*} \rightarrow \mathbf{K}_{\mathcal{B}}(W)$ is defined. Using the universal property of the algebraic tensor product we obtain a unique linear operator $\tilde{\varphi}: V \otimes V^{*} \rightarrow$ $\mathbf{K}_{\mathcal{B}}(W), \tilde{\varphi}\left(x \otimes y^{*}\right)=F_{\phi(x), \phi(y)}$. Since $\phi$ is a $\varphi$-morphism we also have

$$
\begin{aligned}
\tilde{\varphi}\left(x a \otimes y^{*}\right) & =F_{\phi(x a), \phi(y)}=F_{\phi(x) \varphi(a), \phi(y)}=F_{\phi(x), \phi(y) \varphi\left(a^{*}\right)} \\
& =F_{\phi(x), \phi\left(y a^{*}\right)}=\tilde{\varphi}\left(x \otimes a y^{*}\right) .
\end{aligned}
$$

Consequently, we can pass to the quotient i.e. to the modular algebraic tensor product and obtain an unique linear operator $\tilde{\varphi}: V \otimes_{\mathcal{A}} V^{*} \rightarrow \mathbf{K}_{\mathcal{B}}(W)$.

If it is bounded with respect to the Haagerup norm on $V \otimes_{\mathcal{A}} V^{*}$, then we can extend the last map to the desired $\mathrm{C}^{*}$-morphism between the $\mathrm{C}^{*}$-algebras of generalized compact operators. It is sufficient to check the boundedness on positive elements i.e. on $\mathbf{F}_{\mathcal{A}}(V)_{+}$. Using the fact that the completely bounded norm of a $\mathrm{C}^{*}$-morphism equals its usual norm and the formula (see [4]) $\left\|\sum F_{x_{i}, x_{i}}\right\|_{\mathbf{K}_{\mathcal{A}}(V)}=\left\|\left[\left\langle x_{i} \mid x_{j}\right\rangle\right]\right\|_{M_{n}(\mathcal{A})}$ we obtain:

$$
\begin{aligned}
& \left\|\tilde{\varphi}\left(\sum F_{x_{i}, x_{i}}\right)\right\|=\left\|\sum F_{\phi\left(x_{i}\right), \phi\left(x_{i}\right)}\right\|=\left\|\left[\left\langle\phi\left(x_{i}\right) \mid \phi\left(x_{j}\right)\right\rangle\right]\right\|_{M_{n}(\mathcal{B})} \\
& \quad=\left\|\varphi_{n}\left[\left\langle x_{i} \mid x_{j}\right\rangle\right]\right\| \leq\|\varphi\|_{c b}\left\|\left[\left\langle x_{i} \mid x_{j}\right\rangle\right]\right\|=\|\varphi\|\left\|\sum F_{x_{i}, x_{i}}\right\| .
\end{aligned}
$$


Let us finally check that the extension by continuity obtained by the preceding calculation is a $\mathrm{C}^{*}$-morphism:

$$
\begin{aligned}
\tilde{\varphi}\left(\left(\sum_{i} F_{x_{i}, y_{i}}\right)\left(\sum_{j} F_{a_{i}, b_{i}}\right)\right) & =\tilde{\varphi}\left(\sum_{i, j} F_{x_{i}\left\langle y_{i} \mid a_{j}\right\rangle, b_{j}}\right)=\sum_{i, j} F_{\phi\left(x_{i}\left\langle y_{i} \mid a_{j}\right\rangle\right), \phi\left(b_{j}\right)} \\
& =\tilde{\varphi}\left(\sum_{i} F_{x_{i}, y_{i}}\right) \tilde{\varphi}\left(\sum_{j} F_{a_{i}, b_{i}}\right),
\end{aligned}
$$

(so by continuity we have $\tilde{\varphi}\left(K_{1} K_{2}\right)=\tilde{\varphi}\left(K_{1}\right) \tilde{\varphi}\left(K_{2}\right)$ for all $K_{1}, K_{2} \in \mathbf{K}_{\mathcal{A}}(V)$ ), and

$$
\begin{aligned}
\tilde{\varphi}\left(\left(\sum_{i} F_{x_{i}, y_{i}}\right)^{*}\right) & =\tilde{\varphi}\left(\sum_{i} F_{y_{i}, x_{i}}\right)=\sum_{i} F_{\phi\left(y_{i}\right), \phi\left(x_{i}\right)} \\
& =\left(\sum_{i} F_{\phi\left(x_{i}\right), \phi\left(y_{i}\right)}\right)^{*}=\tilde{\varphi}\left(\sum_{i} F_{x_{i}, y_{i}}\right)^{*}
\end{aligned}
$$

(so by continuity we have $\tilde{\varphi}\left(K^{*}\right)=\tilde{\varphi}(K)^{*}$ for all $K \in \mathbf{K}_{\mathcal{A}}(V)$ ).

Now we have $\phi$ (and $\left.\phi^{*}\right), \varphi$ and $\tilde{\varphi}$, so defining

$$
\Phi\left[\begin{array}{ll}
K & x \\
y^{*} & a
\end{array}\right]=\left[\begin{array}{cc}
\tilde{\varphi}(K) & \phi(x) \\
\phi^{*}\left(y^{*}\right) & \varphi(a)
\end{array}\right]
$$

for all $K \in \mathbf{K}_{\mathcal{A}}(V), a \in \mathcal{A}, x, y \in V$ we obtain a linear operator between the linking-algebras which is easily checked to be an $\mathrm{C}^{*}$-morphism.

Above we have obtained the expected result that a morphism of Hilbert $\mathrm{C}^{*}$-modules can be extended to a $\mathrm{C}^{*}$-morphism of the corresponding linkingalgebras. It is natural to pose a more general question: under which conditions can a (bounded) linear operator between two Hilbert $\mathrm{C}^{*}$-modules be extended to a $\mathrm{C}^{*}$-morphism between their linking-algebras? In [13] B. Solel has proven:

Theorem 2.2 (Solel). Every surjective linear isometry $\phi: V \rightarrow W$ between full Hilbert $C^{*}$-modules can be extended to a surjective linear isometry $\Phi: \mathcal{L}_{V} \rightarrow \mathcal{L}_{W}$ of the corresponding linking-algebras mapping the diagonal $\mathbf{K}_{\mathcal{A}}(V) \oplus \mathcal{A}$ of $\mathcal{L}_{V}$ onto the diagonal $\mathbf{K}_{\mathcal{B}}(W) \oplus \mathcal{B}$ of $\mathcal{L}_{W}$. If $\phi$ is a 2-isometry, then $\Phi$ is $C^{*}$-morphism of the form $\Phi=\left[\begin{array}{ll}\tilde{\varphi} & \phi \\ \phi^{*} & \varphi\end{array}\right]$ and the maps $\varphi$ and $\tilde{\varphi}$ are $C^{*}$-isomorphisms.

We shall prove another theorem of similar kind: removing the condition of surjectivity (and 2-isometry) and replacing them by another condition we shall obtain a result for another general situation.

First observe the following simple facts:

Lemma 2.3. For a full Hilbert $C^{*}$-module $V$ and a $\varphi$-morphism $\phi: V \rightarrow$ $W$ of (right) Hilbert $C^{*}$-modules, there is a $C^{*}$-morphism $\tilde{\varphi}: \mathbf{K}_{\mathcal{A}}(V) \rightarrow$ $\mathbf{K}_{\mathcal{B}}(W)$ such that $\phi$ is also a $\tilde{\varphi}$-morphism of left Hilbert $C^{*}$-modules. The 
dual is also true: if $\phi$ is a $\tilde{\varphi}$-morphism of left Hilbert $C^{*}$-modules, there is a $C^{*}$-morphism $\varphi: \mathcal{A} \rightarrow \mathcal{B}$ (defined by $\varphi(\langle x \mid y\rangle)=\langle\phi(x) \mid \phi(y)\rangle$ ) and $\phi$ is a $\varphi$-morphism of right Hilbert $C^{*}$-modules. We shall call such a $\phi$ a $\tilde{\varphi}-\varphi$ morphism.

Proof. The first claim is proven in Proposition 2.1. The dual can be proven in the same way, except that $\mathcal{A}$ and $\mathbf{K}_{\mathcal{A}}(V)$ exchange their roles (and one uses the correspondence $\mathcal{A}=V^{*} \otimes_{h \mathbf{K}_{\mathcal{A}}(V)} V$ which holds for full Hilbert $\mathrm{C}^{*}$-modules $V$ ). It is obvious from the definition of $\tilde{\varphi}$ resp. of $\varphi$ that $\phi$ is a $\tilde{\varphi}-\varphi$-morphism.

Lemma 2.4. If a linear operator $\phi: V \rightarrow W$ can be extended to a $C^{*}$ morphism $\Phi: \mathcal{L}_{V} \rightarrow \mathcal{L}_{W}$ of the form $\Phi=\left[\begin{array}{cc}\tilde{\varphi} & \phi \\ \phi^{*} & \varphi\end{array}\right]$, then $\tilde{\varphi}$ and $\varphi$ are $C^{*}$-morphisms and $\phi$ is a $\tilde{\varphi}-\varphi$-morphism. In particular, $\phi$ is a complete contraction and has the following property: for all $x, y, z \in V$ we have

$$
\phi(x\langle y \mid z\rangle)=\phi(x)\langle\phi(y) \mid \phi(z)\rangle .
$$

Proof. If $\Phi$ is of the above form, by checking its action on elements of the form $\left[\begin{array}{cc}K & 0 \\ 0 & 0\end{array}\right]$ and $\left[\begin{array}{ll}0 & 0 \\ 0 & a\end{array}\right]$ we obtain that $\varphi$ and $\tilde{\varphi}$ are $\mathrm{C}^{*}$-morphisms. It also follows by simple calculation that $\phi$ is a $\tilde{\varphi}-\varphi$-morphism and that property 2.1 holds. Since every $\varphi$-morphism is a complete contraction, so is $\phi$.

From the Solel's theorem is we conclude that every surjective 2-isometry has the property (2.1). The main result of this section is the following:

Theorem 2.5. Let $V$ be a full Hilbert $\mathcal{A}$-module and $W$ a Hilbert $\mathcal{B}$ module. Let $\phi: V \rightarrow W$ be a completely bounded linear operator with the property (2.1). Then there exist (unique) $C^{*}$-morphisms $\varphi: \mathcal{A} \rightarrow \mathcal{B}, \tilde{\varphi}$ : $\mathbf{K}_{\mathcal{A}}(V) \rightarrow \mathbf{K}_{\mathcal{B}}(W)$ and $\Phi: \mathcal{L}_{V} \rightarrow \mathcal{L}_{W}$ such that $\phi$ is a $\varphi-\tilde{\varphi}$-morphism and $\Phi=\left[\begin{array}{cc}\tilde{\varphi} & \phi \\ \phi^{*} & \varphi\end{array}\right]$. If $\phi$ is a complete isometry, all the above $C^{*}$-morphisms are injective. If $\phi$ is surjective and $W$ is full, then all the above $C^{*}$-morphisms are surjective. In particular, any surjective linear isometry of full Hilbert $C^{*}$ modules having the property (2.1) is an isomorphism of Hilbert $C^{*}$-modules.

Proof. By Lemma 2.3, it suffices to show that $\phi$ is a $\varphi$ - or $\tilde{\varphi}$-morphism. We again use the fact that for full modules $\mathcal{A}=V^{*} \otimes_{h \mathbf{K}_{\mathcal{A}}(V)} V$ holds (completely isometrically). Using the universal property of the algebraic tensor product we obtain a linear operator $\varphi: V^{*} \otimes V \rightarrow \mathcal{B}$ from the bilinear operator $\left(x^{*}, y\right) \mapsto\langle\phi(x) \mid \phi(y)\rangle$. To switch to the modular tensor product, we use 
the relation (2.1) and the continuity of $\phi$. We have (for finite sums)

$$
\begin{aligned}
\varphi & \left(\left(x^{*} \sum F_{x_{i}, y_{i}}\right) \otimes y\right)=\varphi\left(\sum\left(y_{i}\left\langle x_{i} \mid x\right\rangle\right)^{*} \otimes y\right) \\
& =\sum\left\langle\phi\left(y_{i}\left\langle x_{i} \mid x\right\rangle\right) \mid \phi(y)\right\rangle=(2.1)=\sum\left\langle\phi\left(y_{i}\right)\left\langle\phi\left(x_{i}\right) \mid \phi(x)\right\rangle \mid \phi(y)\right\rangle \\
& =\sum\left\langle\phi(x) \mid \phi\left(x_{i}\right)\right\rangle\left\langle\phi\left(y_{i}\right) \mid \phi(y)\right\rangle=\sum\left\langle\phi(x) \mid \phi\left(x_{i}\right)\left\langle\phi\left(y_{i}\right) \mid \phi(y)\right\rangle\right\rangle \\
& =(2.1)=\sum\left\langle\phi(x) \mid \phi\left(x_{i}\left\langle y_{i} \mid y\right\rangle\right)\right\rangle=\varphi\left(x^{*} \otimes\left(\sum F_{x_{i}, y_{i}}\right) y\right) .
\end{aligned}
$$

This implies that

$$
\varphi\left(\left(x^{*} K_{\lambda}\right) \otimes y\right)=\varphi\left(x^{*} \otimes K_{\lambda} y\right)
$$

for all $K_{\lambda} \in \mathbf{F}_{\mathcal{A}}(V)$ : If $K=\lim _{\lambda} K_{\lambda}$, then $K^{*}=\lim _{\lambda} K_{\lambda}^{*}$ and $\phi(K x)=$ $\lim _{\lambda} \phi\left(K_{\lambda} x\right)$ (due to continuity of $\left.\phi:\left\|\phi(K x)-\phi\left(K_{\lambda} x\right)\right\| \leq\left\|\left(K-K_{\lambda}\right) x\right\| \rightarrow 0\right)$. As the inner product on a Hilbert $C^{*}$-module is continuous in each of its variables separately, it follows that

$$
\begin{aligned}
& \varphi\left(\left(x^{*} K\right) \otimes y\right)=\left\langle\phi\left(K^{*} x\right) \mid \phi(y)\right\rangle=\lim _{\lambda}\left\langle\phi\left(K_{\lambda}^{*} x\right) \mid \phi(y)\right\rangle= \\
& =\lim _{\lambda}\left\langle\phi(x) \mid \phi\left(K_{\lambda} y\right)\right\rangle=\langle\phi(x) \mid \phi(K y)\rangle=\varphi\left(x^{*} \otimes K y\right) .
\end{aligned}
$$

Passing to the quotient we obtain an operator which we again denote $\varphi$ : $V^{*} \otimes_{\mathbf{K}_{\mathcal{A}}(V)} V \rightarrow \mathcal{B}$. To extend $\varphi$ by continuity to all of $\mathcal{A}$, we have to check the continuity with respect to the (modular) Haagerup norm. Here we need the complete boundedness of $\phi$ :

$$
\left\|\left[\phi\left(x_{i j}\right)\right]\right\|_{n} \leq\|\phi\|_{c b}\left\|\left[x_{i j}\right]\right\|_{n}
$$

i.e.

$$
\left\|\left[\sum_{k=1}^{n}\left\langle\phi\left(x_{k i}\right) \mid \phi\left(x_{k j}\right)\right\rangle\right]\right\| \leq\|\phi\|_{c b}^{2}\left\|\left[\sum_{k=1}^{n}\left\langle x_{k i} \mid x_{k j}\right\rangle\right]\right\|
$$

for all $n \in \mathbf{N}$ and $x_{i j} \in V$. Taking

$$
\left[x_{i j}\right]=\left[\begin{array}{cccc}
x_{1} & 0 & \ldots & 0 \\
\vdots & \vdots & & \vdots \\
x_{n} & 0 & \ldots & 0
\end{array}\right]
$$

with arbitrary $x_{1}, \ldots, x_{n} \in V$ we get

$$
\begin{aligned}
\left\|\left[\sum_{k=1}^{n}\left\langle\phi\left(x_{k i}\right) \mid \phi\left(x_{k j}\right)\right\rangle\right]\right\| & =\left\|\left[\begin{array}{cccc}
\sum\left\langle\phi\left(x_{k}\right) \mid \phi\left(x_{k}\right)\right\rangle & 0 & \ldots & 0 \\
\vdots & \vdots & & \vdots \\
0 & 0 & \ldots & 0
\end{array}\right]\right\| \\
& \leq\|\phi\|_{c b}^{2}\left\|\left[\begin{array}{cccc}
\sum\left\langle x_{k} \mid x_{k}\right\rangle & 0 & \ldots & 0 \\
\vdots & \vdots & & \vdots \\
0 & 0 & \ldots & 0
\end{array}\right]\right\|
\end{aligned}
$$


or finally

$$
\begin{aligned}
& \left\|\varphi\left(\sum x_{k}^{*} \otimes_{\mathbf{K}_{\mathcal{A}}(V)} x_{k}\right)\right\|=\left\|\sum\left\langle\phi\left(x_{k}\right) \mid \phi\left(x_{k}\right)\right\rangle\right\| \\
& \quad \leq\|\phi\|_{c b}^{2}\left\|\sum\left\langle x_{k} \mid x_{k}\right\rangle\right\|=\|\phi\|_{c b}^{2}\left\|\sum x_{k}^{*} \otimes_{\mathbf{K}_{\mathcal{A}}(V)} x_{k}\right\| .
\end{aligned}
$$

This shows that $\varphi$ is continuous on positive elements in $V^{*} \otimes \mathbf{K}_{\mathcal{A}}(V) V$, which are dense in $\mathcal{A}_{+}$because $V$ is full by assumption. As every element in a $\mathrm{C}^{*}-$ algebra is a linear combination of four positive elements, $\varphi$ can be extended by continuity to $\mathcal{A}$ and it is easily verified that this extension is a $\mathrm{C}^{*}$-morphism. By Proposition 2.1 we now obtain $\tilde{\varphi}$ i $\Phi$.

An analogy of the well-known theorem that every $\mathrm{C}^{*}$-morphism is a (complete) contraction is the following:

COROllary 2.6. Every completely bounded operator between Hilbert $C^{*}$ modules $\phi: V \rightarrow W$ with $V$ full and which satisfies (2.1) is a complete contraction.

Proof. By the above theorem, $\phi$ is a $\varphi$-morphism, and as such it is a complete contraction.

Note that according to the above corollary one can define a Hilbert $\mathrm{C}^{*}$ morphism without referring to the supporting $\mathrm{C}^{*}$-morphism: if $\phi: V \rightarrow W$ is a bounded linear operator (and $V$ a full module), we call $\phi$ a Hilbert $\mathrm{C}^{*}$ morphism if it has property (2.1).

REMARK 2.7. With a different argument than above, one can prove that a bounded operator satisfying (2.1) is contractive, even if the module is not full. For this it is necessary to note that for all elements $x$ of a general Hilbert $\mathrm{C}^{*}$-module the formula

$$
\|\langle x \mid x\rangle x\|=\|x\|^{3}
$$

is valid: since $\langle x \mid x\rangle$ is selfadjoint, one has

$$
\begin{aligned}
\|\langle x \mid x\rangle x\|^{2} & =\|\langle\langle x \mid x\rangle x \mid\langle x \mid x\rangle x\rangle\|=\|\langle x \mid x\rangle\langle x \mid x\rangle\langle x \mid x\rangle\| \\
& =\|\langle x \mid x\rangle\|^{3}=\|x\|^{6} .
\end{aligned}
$$

Accordingly, for a bounded operator $\phi$ satisfying (2.1) one has

$$
\begin{aligned}
\|\phi(x)\|^{3} & =\|\langle\phi(x) \mid \phi(x)\rangle \phi(x)\|=\|\phi(\langle x \mid x\rangle x)\| \\
& \leq\|\phi\| \cdot\|\langle x \mid x\rangle x\|=\|\phi\| \cdot\|x\|^{3}
\end{aligned}
$$

from where $\|\phi\| \leq 1$ easily follows.

The question remains if the condition (2.1) is necessary for the above constructions? By Solel's results, for a surjective 2-isometry $\phi$ it is not a necessary condition. It can also be removed in a slightly more general case: For a 2-isometry $\phi: V \rightarrow W$ such that its range $\phi(V)$ is a Hilbert $\mathrm{C}^{*}$ submodule of $W$, one can apply the Solel theorem to $\phi: V \rightarrow \phi(V)$ and 
obtain $\Phi: \mathcal{L}_{V} \rightarrow \mathcal{L}_{\phi(V)} \subseteq \mathcal{L}_{W}$ of the form $\left[\begin{array}{cc}\tilde{\varphi} & \phi \\ \phi^{*} & \varphi\end{array}\right]$ such that $\phi$ is a $\tilde{\varphi}-\varphi$-morphism.

But the result from Theorem 2.5 covers some cases which cannot be obtained by Solel's theorem because even for a complete isometry between Hilbert $\mathrm{C}^{*}$-modules it is possible that the range is not a Hilbert $\mathrm{C}^{*}$-module.

ExAmple 2.8. Let $V=W=\mathcal{A}$ and let $\phi: \mathcal{A} \rightarrow \mathcal{A}$ be a (completely isometric) completely positive operator. As closed submodules of $\mathcal{A}$ are precisely the right (closed) ideals in $\mathcal{A}$, and as it is known that a completely positive operator generally doesn't map ideals onto ideals, it follows that $\phi(\mathcal{A})$ doesn't have to be a Hilbert $\mathrm{C}^{*}$-submodule of $\mathcal{A}$.

It is also not possible to replace the condition (2.1) by restricting the theorem to complete isometries because there are complete isometries which don't have this property:

Example 2.9. Define $\phi: \mathcal{A} \rightarrow \mathcal{A}$ by $\phi(a)=v^{*} a v$, where $v$ is an isometry which isn't a coisometry. Then $\phi_{n}\left[a_{i j}\right]=\operatorname{diag}\left(v^{*}\right)\left[a_{i j}\right] \operatorname{diag}(v)$ and

$$
\operatorname{diag}(v)=\left(\begin{array}{ccc}
v & & 0 \\
& \ddots & \\
0 & & v
\end{array}\right)
$$

is also an isometry which isn't a coisometry for all $n \in \mathbf{N}$. This means that $\phi$ is a complete isometry if it is an isometry. It is obviously a contraction. Because $v$ is isometric, we have

$$
\|a\|=\sup _{\|\xi\| \leq 1}(a \xi \mid \xi) \leq \sup _{\|v \xi\| \leq 1}(a v \xi \mid v \xi)=\sup _{\|v \xi\| \leq 1}\left(v^{*} a v \xi \mid \xi\right)=\|\phi(a)\|
$$

so $\phi$ is a (complete) isometry.

But $\phi$ of this form can fail to hold (2.1). Taking e.g. $\mathcal{A}=\mathbf{B}\left(l_{2}\right)$ and $v$ to be an unilateral shift, for $a \in \mathcal{A}$ defined by $a e_{1}=a e_{2}=e_{2}, a e_{i}=0$ for $i \geq 3$ (by $e_{i}$ we denote the canonical basis of $\left.l_{2}\right)$, we have $\phi\left(a a^{*} a\right) e_{1}=2 e_{1} \neq e_{1}=$ $\phi(a) \phi(a)^{*} \phi(a) e_{1}$.

\section{Hilbert $\mathrm{C}^{*}$-extensions of operator sPACES AND EXTEnsions of \\ HilberT $\mathrm{C}^{*}$-MODUles}

In [5] D. P. Blecher introduces extensions of operator spaces which are Hilbert $\mathrm{C}^{*}$-modules, an order relation on the set of all such extensions and proves that there is a minimal Hilbert $\mathrm{C}^{*}$-extension of a given operator space (and in [6] proves there is a maximal one). The definition is as follows:

Definition 3.1. A Hilbert $C^{*}$-extension of an operator space $X$ is an ordered pair $(V, i)$, where $V$ is a Hilbert $C^{*}$-module, and $i: X \rightarrow V$ is a complete isometry such that $i(X)$ generates the linking algebra $\mathcal{L}_{V}$. 
Since every Hilbert $\mathrm{C}^{*}$-module is an operator space, the natural question to ask is what happens if $X$ is already a $\left(\right.$ full $\left.{ }^{3}\right)$ Hilbert $\mathrm{C}^{*}$-module. Examining the definition more carefully, it is obvious that in this case the Hilbert $\mathrm{C}^{*}$ extension is unique - the only full Hilbert $\mathrm{C}^{*}$-module generating the same linking algebra is the module we started with.

As described in the introduction, there is a natural extension concept for Hilbert $\mathrm{C}^{*}$-modules. The objective of this section is to provide a connection between Blecher's Hilbert $\mathrm{C}^{*}$-extensions of operator spaces and the $\mathrm{C}^{*}$-extensions of Hilbert $\mathrm{C}^{*}$-modules as introduced by D. Bakić and B. Guljaš $([2])$. In order to obtain such a connection, it is necessary to generalize Blecher's definition in a way which will ensure that taking an operator space which is also o Hilbert $\mathrm{C}^{*}$-module one generally gets more than one extension. Since the reason why Blecher's definition doesn't give more than one extension for a Hilbert $\mathrm{C}^{*}$-module is in the condition that the image of the operator space in its extension generates the whole linking-algebra, we will alter the definition so that we'll require that the image generates only a natural subobject of the linking-algebra: an ideal. So we'll use the following generalization of Blecher's definition:

Definition 3.2. A Hilbert $\mathrm{C}^{*}$-extension of an operator space $X$ is a triple $(W, \mathcal{B}, \phi)$, where $W$ is a Hilbert $\mathcal{B}$-module and $\phi: X \rightarrow W$ is a complete isometry such that the image $\phi(X)$ generates a $C^{*}$-algebra which is an ideal in the linking-algebra $\mathcal{L}_{W}$.

Note that the requirement that $\phi(X)$ generates an ideal in the linkingalgebra can be replaced by the equivalent condition that it is an ideal submodule of $W$.

Since ideal submodules generated by essential ideals are in 1-1-correspondence with essential ideals in the linking-algebra, it is also possible to define an essential Hilbert $C^{*}$-extension of an operator space $X$ as an Hilbert $\mathrm{C}^{*}$ extension satisfying the additional property that $\phi(X)$ generates an essential ideal in the linking-algebra.

To obtain the 1 - 1-correspondence between Hilbert $\mathrm{C}^{*}$-extensions in the sense of Definition 3.2 and $\mathrm{C}^{*}$-extensions in the sense of Bakić and Guljaš, the first step is the following:

Proposition 3.3. Every (essential) $C^{*}$-extension of a full Hilbert $C^{*}$ module is an (essential) Hilbert $C^{*}$-extension of the module.

Proof. If $V$ is a full Hilbert $\mathrm{C}^{*}$-module and $(W, \mathcal{B}, \phi, \varphi)$ a $\mathrm{C}^{*}$-extension of $V$, then by definition $\phi(V)$ generates an ideal in $\mathcal{L}_{W}$. Since every isometric morphism of Hilbert $\mathrm{C}^{*}$-modules is completely isometric, $(W, \mathcal{B}, \phi)$ is a Hilbert $\mathrm{C}^{*}$-extension of $V$. It is also obvious that an essential $\mathrm{C}^{*}$-extension is an essential Hilbert $\mathrm{C}^{*}$-extension of $V$.

\footnotetext{
${ }^{3}$ Note that we considered $\mathrm{C}^{*}$-extensions only for full modules.
} 
Using the results of the previous section it is now possible to prove that the complete isometry $\phi$ in the definition of a Hilbert $\mathrm{C}^{*}$-extension is a morphism of Hilbert $\mathrm{C}^{*}$-modules. This will imply that there is an $1-1$-correspondence between the two definitions of extensions (for a given full Hilbert $\mathrm{C}^{*}$-module).

Theorem 3.4. $(W, \mathcal{B}, \phi)$ is a Hilbert $C^{*}$-extension of a full Hilbert $\mathcal{A}$ module $V$ if and only if $(W, \mathcal{B}, \phi, \varphi)$ is a $C^{*}$-extension of $V$, where $\varphi: \mathcal{A} \rightarrow \mathcal{B}$ is an injective $C^{*}$-morphism required for the $C^{*}$-extension (i.e. such that $\phi$ is a $\varphi$-morphism).

Proof. Let $(W, \mathcal{B}, \phi)$ be a Hilbert $\mathrm{C}^{*}$-extension of a full Hilbert $\mathcal{A}$ module $V$. By definition $\phi$ is a complete isometry and $\phi(V)$ is an ideal submodule of $W$, generated by an ideal $\mathcal{I}$ in $\mathcal{B}$ i.e. $\phi(V)=W_{\mathcal{I}}$. Without loss of generality we may assume that $W_{\mathcal{I}}$ is a full module. This means we have a surjective complete isometry $\phi: V \rightarrow W_{\mathcal{I}}$. By Solel's theorem it induces $\mathrm{C}^{*}$-isomorphisms $\varphi: \mathcal{A} \rightarrow \mathcal{I} \subseteq \mathcal{B}, \tilde{\varphi}: \mathbf{K}_{\mathcal{A}}(V) \rightarrow \mathbf{K}_{\mathcal{I}}\left(W_{\mathcal{I}}\right) \subseteq \mathbf{K}_{\mathcal{B}}(W)$ and $\Phi: \mathcal{L}_{V} \rightarrow \mathcal{L}_{W_{\mathcal{I}}} \subseteq \mathcal{L}_{W}$. So $\varphi: \mathcal{A} \rightarrow \mathcal{B}$ is the required injective $\mathrm{C}^{*}$-morphism with an ideal $\mathcal{I}$ as its image, corresponding to the image $\phi(V)=W_{\mathcal{I}}$.

As the corresponding $\mathrm{C}^{*}$-morphism $\varphi$ of an morphism $\phi$ of Hilbert $\mathrm{C}^{*}$ modules is unique, the mapping $(W, \mathcal{B}, \phi, \varphi) \mapsto(W, \mathcal{B}, \phi)$ is injective, and by the preceding proposition it is surjective.

Blecher defined an order relation for Hilbert $\mathrm{C}^{*}$-extensions of a given operator space, which can be applied to our generalized definition as well:

Definition 3.5. If $\left(W_{1}, \mathcal{B}_{1}, \phi_{1}\right)$ and $\left(W_{2}, \mathcal{B}_{2}, \phi_{2}\right)$ are two Hilbert $C^{*}$ extensions of an operator space $X$, we define $\left(W_{1}, \mathcal{B}_{1}, \phi_{1}\right) \leq\left(W_{2}, \mathcal{B}_{2}, \phi_{2}\right)$ if there is a surjective complete contraction $T: W_{2} \rightarrow W_{1}$ such that $T \phi_{2}=\phi_{1}$ and there is a surjective $C^{*}$-morphism $\hat{\tau}: \mathcal{L}_{W_{2}} \rightarrow \mathcal{L}_{W_{1}}$ such that $\hat{\tau}\left[\begin{array}{ll}0 & x \\ 0 & 0\end{array}\right]=\left[\begin{array}{cc}0 & T(x) \\ 0 & 0\end{array}\right]$ for all $x \in W_{2}$.

For $\mathrm{C}^{*}$-extensions, a sensible definition of the order relation (inspired by the above in order to ensure that the correspondence between Hilbert $\mathrm{C}^{*}$ extensions and $\mathrm{C}^{*}$-extensions is order preserving, but ensuring the maximal essential extension to remain maximal in this order) is:

Definition 3.6. Let $\left(W_{1}, \mathcal{B}_{1}, \phi_{1}, \varphi_{1}\right)$ and $\left(W_{2}, \mathcal{B}_{2}, \phi_{2}, \varphi_{2}\right)$ be two $C^{*}$ extensions of a Hilbert $C^{*}$-module $V$. We define $\left(W_{1}, \mathcal{B}_{1}, \phi_{1}, \varphi_{1}\right) \leq\left(W_{2}, \mathcal{B}_{2}\right.$, $\left.\phi_{2}, \varphi_{2}\right)$ if there is a surjective $C^{*}$-morphism $\tau: \mathcal{B}_{2} \rightarrow \mathcal{B}_{1}$ and a surjective $\tau$-morphism $T: W_{2} \rightarrow W_{1}$ such that $\tau \varphi_{2}=\varphi_{1}$ and $T \phi_{2}=\phi_{1}$.

Proposition 3.7. The bijection between Hilbert $C^{*}$-extensions and $C^{*}$ extensions of a given full Hilbert $C^{*}$-module from the Theorem 3.4 is order preserving. 
Proof. Let $\left(W_{1}, \mathcal{B}_{1}, \phi_{1}, \varphi_{1}\right) \leq\left(W_{2}, \mathcal{B}_{2}, \phi_{2}, \varphi_{2}\right)$ be two $\mathrm{C}^{*}$-extensions of a full Hilbert $\mathcal{A}$-module $V$. By definition, there is a surjective $\mathrm{C}^{*}$-morphism $\tau: \mathcal{B}_{2} \rightarrow \mathcal{B}_{1}$ and a surjective $\tau$-morphism $T: W_{2} \rightarrow W_{1}$ such that $\tau \varphi_{2}=\varphi_{1}$ and $T \phi_{2}=\phi_{1}$. The induced $\mathrm{C}^{*}$-morphism of the linking-algebras, denote it by $\hat{\tau}$, is obviously surjective (because, by its definition, the induced morphism $\tilde{\varphi}$ between the $\mathrm{C}^{*}$-algebras of compact operators is surjective if $\phi$ is). Accordingly $\left(W_{1}, \mathcal{B}_{1}, \phi_{1}\right) \leq\left(W_{2}, \mathcal{B}_{2}, \phi_{2}\right)$.

If $\left(W_{1}, \mathcal{B}_{1}, \phi_{1}\right) \leq\left(W_{2}, \mathcal{B}_{2}, \phi_{2}\right)$ are two Hilbert $\mathrm{C}^{*}$-extensions of $V$ and $T: W_{2} \rightarrow W_{1}$ the corresponding surjective complete contraction with the property $T \phi_{2}=\phi_{1}$ and if $\hat{\tau}: \mathcal{L}_{2} \rightarrow \mathcal{L}_{1}$ is the surjective $\mathrm{C}^{*}$-morphism with the property $\hat{\tau}\left[\begin{array}{ll}0 & x \\ 0 & 0\end{array}\right]=\left[\begin{array}{cc}0 & T(x) \\ 0 & 0\end{array}\right]$, then $T$ must satisfy the property (2.1):

$$
\begin{aligned}
& {\left[\begin{array}{cc}
0 & T(x\langle y \mid z\rangle) \\
0 & 0
\end{array}\right]=\hat{\tau}\left[\begin{array}{cc}
0 & x\langle y \mid z\rangle \\
0 & 0
\end{array}\right]} \\
& \quad=\hat{\tau}\left(\left[\begin{array}{ll}
0 & x \\
0 & 0
\end{array}\right]\left[\begin{array}{cc}
0 & 0 \\
y^{*} & 0
\end{array}\right]\left[\begin{array}{ll}
0 & z \\
0 & 0
\end{array}\right]\right) \\
& \quad=\hat{\tau}\left[\begin{array}{ll}
0 & x \\
0 & 0
\end{array}\right] \hat{\tau}\left[\begin{array}{cc}
0 & 0 \\
y^{*} & 0
\end{array}\right] \hat{\tau}\left[\begin{array}{cc}
0 & z \\
0 & 0
\end{array}\right] \\
& =\left[\begin{array}{cc}
0 & T x \\
0 & 0
\end{array}\right]\left[\begin{array}{cc}
0 & 0 \\
(T y)^{*} & 0
\end{array}\right]\left[\begin{array}{cc}
0 & T z \\
0 & 0
\end{array}\right] \\
& =\left[\begin{array}{cc}
0 & T x\langle T y \mid T z\rangle \\
0 & 0
\end{array}\right] .
\end{aligned}
$$

By Theorem 2.5 there is a $\mathrm{C}^{*}$-morphism $\tau: \mathcal{B}_{2} \rightarrow \mathcal{B}_{1}$ such that $T$ is a $\tau$ morphism. Because of the 1-1-correspondence between the two notions of extensions, there exist injective $\varphi_{i}: \mathcal{A} \rightarrow \mathcal{B}_{i}$ such that $\phi_{i}$ is a $\varphi_{i}$-morphism (for $i=1,2)$. $V$ is a full module, so $\varphi_{1}=\tau \varphi_{2}$. By assumption, $\hat{\tau}$ is surjective, and $\tau$ is (by construction) its lower right corner, so $\tau$ is a surjective $\mathrm{C}^{*}$-morphism. This means that $\left(W_{1}, \mathcal{B}_{1}, \phi_{1}, \varphi_{1}\right) \leq\left(W_{2}, \mathcal{B}_{2}, \phi_{2}, \varphi_{2}\right)$.

As noted before the Definition 3.6, this definition not only ensures the order correspondence between the two notions of extensions, but is also chosen in such a way that the maximal essential $\mathrm{C}^{*}$-extension $\mathcal{M}(V)=\mathbf{B}_{\mathcal{A}}(\mathcal{A}, V)$ of a given full Hilbert $\mathrm{C}^{*}$-module $V$ (which is a generalization of the multiplier $\mathrm{C}^{*}$ algebra as noted in the introduction) is maximal in this order. This shall be proven as the final result of this paper. In the original proof of maximality of $\mathcal{M}(V)$ another order was used, so this is not an obvious result. The originally used order between $\mathrm{C}^{*}$-extensions of a given full module $V$ is defined by $\left(W_{1}, \mathcal{B}_{1}, \phi_{1}, \varphi_{1}\right) \leq\left(W_{2}, \mathcal{B}_{2}, \phi_{2}, \varphi_{2}\right)$ if there is an injective $\mathrm{C}^{*}$-morphism $\varphi$ : $\mathcal{B}_{1} \rightarrow \mathcal{B}_{2}$ and a $\varphi$-morphism $\phi: W_{1} \rightarrow W_{2}$ such that $\varphi \varphi_{1}=\varphi_{2}$ and $\phi \phi_{1}=\phi_{2}$. Our definitions of order relations use surjective morphisms, so it is not obvious that the maximal (with respect to the just described order) essential extension 
is maximal in our sense. The morphisms $\gamma: \mathcal{A} \rightarrow \mathcal{M}(\mathcal{A})$ and $\Gamma: V \rightarrow \mathcal{M}(V)$ are the canonical embeddings.

Proposition 3.8. $(\mathcal{M}(V), \mathcal{M}(\mathcal{A}), \Gamma, \gamma)$ is a maximal essential $C^{*}$-extension of a full Hilbert $\mathcal{A}$-module $V$, with respect to the order defined in Definition 3.6.

Proof. Let $(W, \mathcal{B}, \phi, \varphi)$ be any essential $\mathrm{C}^{*}$-extension of $V$. Suppose $(W, \mathcal{B}, \phi, \varphi) \geq(\mathcal{M}(V), \mathcal{M}(\mathcal{A}), \Gamma, \gamma)$ i.e. there is a surjective $\mathrm{C}^{*}$-morphism $\rho: \mathcal{B} \rightarrow \mathcal{M}(\mathcal{A})$ such that $\rho \varphi=\gamma$ and a surjective $\rho$-morphism $R: W \rightarrow \mathcal{M}(V)$ such that $R \phi=\Gamma$. As $\varphi(\mathcal{A})$ is an essential ideal in $\mathcal{B}$, and $\operatorname{Ker}(\rho)$ is a closed ideal in $\mathcal{B}$, we have $\varphi(\mathcal{A}) \cap \operatorname{Ker}(\rho) \neq\{0\}$ if $\operatorname{Ker}(\rho) \neq\{0\}$. But if $a \in \mathcal{A}$ is such that $\varphi(a) \in \varphi(\mathcal{A}) \cap \operatorname{Ker}(\rho)$, then $0=\rho(\varphi(a))=\gamma(a)$. As $\gamma$ is injective, it follows that $a=0$ i.e. $\operatorname{Ker}(\rho)=\{0\}$. So $\rho$ is a $\mathrm{C}^{*}$-isomorphism. Accordingly, $R$ is injective so $\mathcal{B}$ is isomorphic to $\mathcal{M}(\mathcal{A})$ and $W$ to $\mathcal{M}(V)$.

Corollary 3.9. $(\mathcal{M}(V), \mathcal{M}(\mathcal{A}), \Gamma)$ is a maximal essential Hilbert $C^{*}$ extension of a full Hilbert $\mathcal{A}$-module $V$.

Proof. As any injective order preserving mapping preserves maximal elements, the bijective order preserving correspondence between Hilbert $\mathrm{C}^{*}$ extensions and $\mathrm{C}^{*}$-extensions of $V$ provides the result.

\section{ACKNowledgements.}

This paper is a part of the author's Ph.D. dissertation at the University of Zagreb. I wish to express gratitude to my advisor D. Bakić for his guidance and encouragement and to professor B. Guljaš for his helpful comments.

I also wish to thank the referee for his helpful suggestions, in particular for pointing out the other approach to showing contractivity of a bounded operator satisfying (2.1), as described in Remark 2.7.

\section{REFERENCES}

[1] D. Bakić and B. Guljaš, On a class of module maps of Hilbert $C^{*}$-modules, Math. Commun. 7 (2002), 177-194.

[2] D. Bakić and B. Guljaš, Extensions of Hilbert $C^{*}$-modules, Houston Math. J. 30 (2004), 537-558.

[3] D. Bakić and B. Guljaš, Extensions of Hilbert $C^{*}$-modules II, Glasnik Mat. 38 (2003), 341-357.

[4] D.P. Blecher, A new approach to Hilbert $C^{*}-$ modules, Math. Ann. 307 (1997), 253290.

[5] D.P. Blecher, The Shilov boundary of an operator space and the characterization theorems, J. Funct. Anal. 182 (2001), 280-343.

[6] D.P. Blecher, Modules over operator algebras, and the maximal $C^{*}$-dilation, J. Funct. Anal. 169 (1999), 251-288.

[7] L.G. Brown, J.A. Mingo and N.-T. Shen, Quasi-multipliers and embeddings of Hilbert $C^{*}$-bimodules, Can. J. Math. 46 (1994), 1150-1174.

[8] I. Kaplansky, Modules over operator algebras, Amer. J. Math. 75 (1953) 839-858. 
[9] E.C. Lance, Hilbert $C^{*}$-modules. A toolkit for operator algebraists, London Mathematical Society Lecture Note Series 210, Cambridge University Press, 1995.

[10] W.L. Paschke, Inner product modules over $B^{*}$-algebras, Trans. Amer. Math. Soc. 182 (1973), 443-468.

[11] V.I. Paulsen, Completely bounded maps and dilations, Pitman Res. Notes in Math. $146,1986$.

[12] M.A. Rieffel, Induced representations of $C^{*}$-algebras, Adv. Math. 13 (1974), 176-257.

[13] B. Solel, Isometries of Hilbert $C^{*}$-modules, Trans. Amer. Math. Soc. 353 (2001), 4637-4660.

F.M. Brückler

Department of Mathematics

University of Zagreb

10000 Zagreb

Croatia

E-mail: bruckler@math.hr

Received: 14.11.2003.

Revised: 01.03.2004. 